\title{
TIME-RESOLVED EMISSION SPECTROSCOPY OF ATOMIC AND MOLECULAR SPECIES IN LASER-INDUCED PLASMA
}

\author{
C.G. PARIGGER ${ }^{1 *}$, G. GAUTAM ${ }^{2}$, C.M. HELSTERN ${ }^{1}$ \\ ${ }^{1}$ University of Tennessee Space Institute, Center for Laser Applications, \\ 411 B.H. Goethert Parkway, Tullahoma, TN 37388-9700, USA \\ ${ }^{2}$ Fort Peck Community College, 605 Indian Avenue, Poplar, MT 59255, USA \\ *Corresponding author E-mail: cparigge@ tennessee.edu (C.G. Parigger)
}

\begin{abstract}
This work examines atomic and molecular signatures in laser-induced plasma in standard ambient temperature and pressure environments, including background contributions to the spectra that depend on the laser pulse-width. Investigations include solids, gases, and nano-particles. Abel inversions of measured line-of-sight data reveal insight into the radial plasma distribution. For nominal 6 nanosecond laser pulses and for pulse-energies in the range of 100 to 800 milli-Joules, expansion dynamics and turbulence due to shock phenomena are elucidated to address local equilibrium details that are frequently assumed in spatially averaged emission spectroscopy. Chemical equilibrium computations reveal temperature dependence of selected plasma species. Specific interests include atomic hydrogen $(\mathrm{H})$ and cyanide $(\mathrm{CN})$. The atomic $\mathrm{H}$ spectra, collected following optical breakdown in ultra-high-pure hydrogen and 9:1 mixtures of ultra-pure hydrogen and nitrogen gases, indicate spherical shell structures and isentropic expansion of the plasma kernel over and above the usual shockwave. The recombination radiation of $\mathrm{CN}$ emanates within the first 100 nanoseconds for laser-induced breakdown in a 1:1 $\mathrm{CO}_{2}: \mathrm{N}_{2}$ gas mixture when using nanosecond laser pulses to create the micro-plasma. The micro-plasma is generated using $1064 \mathrm{~nm}, 150 \mathrm{~mJ}, 6 \mathrm{~ns}$ Q-switched Nd:YAG laser radiation. Measurements of the optical emission spectra utilize a 0.64 m Czerny-Turner type spectrometer and an intensified charge-coupled device.
\end{abstract}

Keywords: Laser-induced plasma; atomic and molecular spectroscopy; Abel inversion; hydrogen; cyanogen;

\section{INTRODUCTION}

Analysis of atomic species traditionally utilizes atomic emission spectroscopy of flames, plasmas, arcs, or sparks for quantification of elements in the sample. However, elemental composition studies frequently apply laserinduced breakdown spectroscopy (LIBS) in a variety of environments and with extensions to molecular characterizations of solids, liquids, and gases ${ }^{1-3}$. Investigations of laser-induced hydrogen plasmas serve the purpose of addressing fundamental aspects of time-resolved emission spectroscopy and associated dynamic processes following optical breakdown. Measurements of hydrogen plasmas allow one to determine important plasma parameters such as excitation electron density and temperature. Usually one measures the width of Balmer series lines of hydrogen that occur in the visible spectrum for electron density determination, and the integrated ratio of these lines or with respect to the continuum. Optical emission spectroscopy (OES) records indicate as well the presence of molecular species that can elucidate sample composition or interaction processes in the ambient atmosphere ${ }^{3-5}$. Typically, atomic hydrogen lines, $\mathrm{C}_{2}$ Swan bands and cyanide $(\mathrm{CN})$ violet system show in various applications $^{5,6}$ of laser-induced OES such as in experiments with hydrocarbons in air. Applications include laser ablation molecular isotope spectrometry ${ }^{7}$, combustion analyses ${ }^{8}$, and plant or medical diagnostics ${ }^{9-11}$. Detection methods of $\mathrm{CN}$ in the medical field include optical methods, electrochemical methods, mass- spectrometry, gas 
chromatography, and quartz crystal mass monitors ${ }^{12}$. These methods are sufficient for detection of cyanide, yet molecular emission spectroscopy allows one to measure composition with a minimally invasive approach.

This work reports selected results from experiments using nanosecond laser spectroscopy of hydrogen plasma ${ }^{13}$ and of $\mathrm{CN}$ molecular emission spectroscopy ${ }^{14}$. The $\mathrm{CN}$ recombination radiation occurs within the first 100 nanoseconds for laser-induced breakdown in 1:1 $\mathrm{CO}_{2}: \mathrm{N}_{2}$ gas mixtures. Aspects of the analysis include Abel inversions ${ }^{15-17}$ and computational modeling ${ }^{5}$ of the plasma. Analysis of asymmetric plasma expansion would require Radon inverse transformations ${ }^{18}$. Use of a chemical equilibrium code ${ }^{19}$ allows one to predict species distribution as function of temperature and pressure and to investigate local thermodynamic equilibrium. Experiments with femtosecond laser pulses reveal advantages for laser ablation ${ }^{10,11}$ with direct surface interaction LIBS, but without the type of laser-supported plasma encountered with nanosecond pulses.

\section{EXPERIMENTAL DETAILS}

The experimental arrangement consists of a set of components typical for time-resolved, laser-induced optical emission spectroscopy ${ }^{13,14}$, or nanosecond laser-induced breakdown spectroscopy (LIBS). Primary instrumentations include a Q-switched Nd:YAG device (Quantel model Q-smart 850) that is operated at the fundamental wavelength of 1064-nm to produce full-width-at-half-maximum 6-ns laser radiation with an energy of $850 \mathrm{~mJ}$ per pulse, a laboratory type Czerny-Turner spectrometer (Jobin Yvon model HR 640) with a $0.64 \mathrm{~m}$ focal length and equipped with a 1200 grooves/mm grating, an intensified charge coupled device (Andor Technology model iStar DH334T-25U-03) for recording of temporally and spatially resolved spectral data, a laboratory chamber or cell with inlet and outlet ports together with a vacuum system, electronic components for synchronization, and various optical elements for beam shaping, steering, and focusing.

A singlet lens (Thorlabs model LA1509-C) accomplishes with f/5 focusing the generation of the optical breakdown micro-plasma in a chamber that contains the 1:1 $\mathrm{CO}_{2}: \mathrm{N}_{2}$ atmospheric gas mixture (Airgas ultra-high purity $\mathrm{N}_{2}$ and research grade $\mathrm{CO}_{2}$ ). Beam-splitters and apertures attenuate the energy/pulse from 850 to $150 \mathrm{~mJ}$ for the $\mathrm{CN}$ experiments. In air breakdown shadow-graph visualizations, a fused silica plano-convex lens (Thorlabs model LA4545) focuses the laser beam with f/10 optics. Figure 1 illustrates computed radial distributions ${ }^{5}$ for the Thorlabs LA4545 lens for focusing with f/5 optics. The peak irradiance distributions are computed for $850 \mathrm{~mJ}, 6$ $\mathrm{ns}, 1064 \mathrm{~nm}$ radiation. The tighter $\mathrm{f} / 5$ focusing reveals about one order of magnitude (or by a factor of $2^{3}$ ) smaller focal volume than that obtained for $\mathrm{f} / 10$ focusing.

(a)

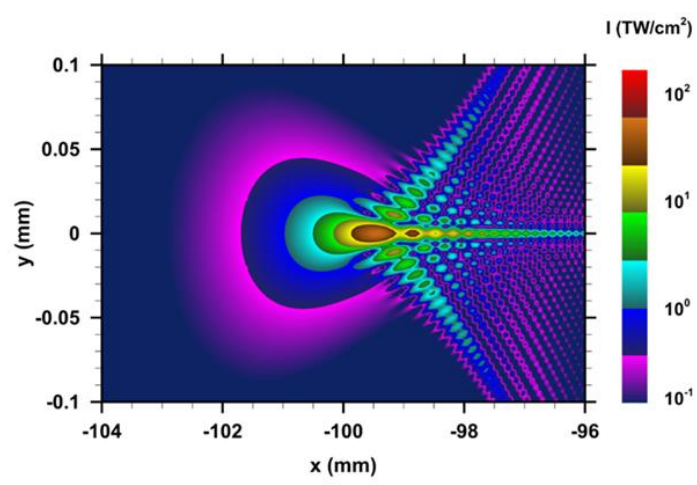

(b)

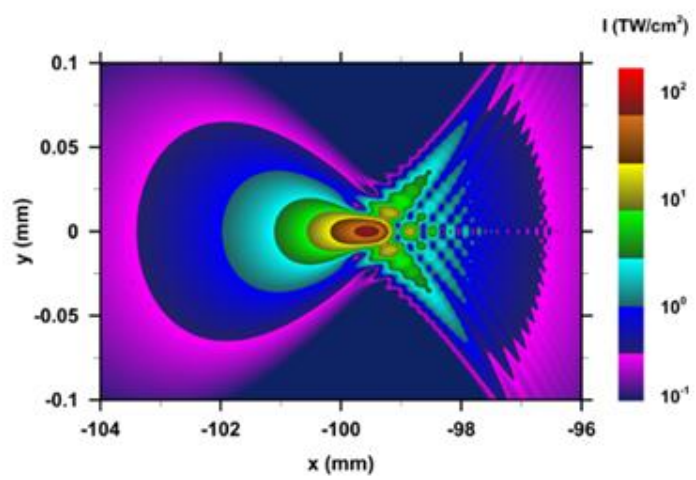

Figure 1: Spatial distribution of 1064-nm, (a) f/5 (b) f/10 focusing with the Thorlabs LA4545, 100 mm focal length lens. 
Captured shadow-graphs of the breakdown plasma serve the purpose of visualizing the plasma $\operatorname{expansion}^{20}$. Figure 2 illustrates typical shadowgraphs recorded in standard ambient temperature and pressure laboratory air. The figures reveal vertical stagnation layers that originate from multiple breakdown sites as indicated in the computed focal intensity distribution (see Fig. 1).

(a)

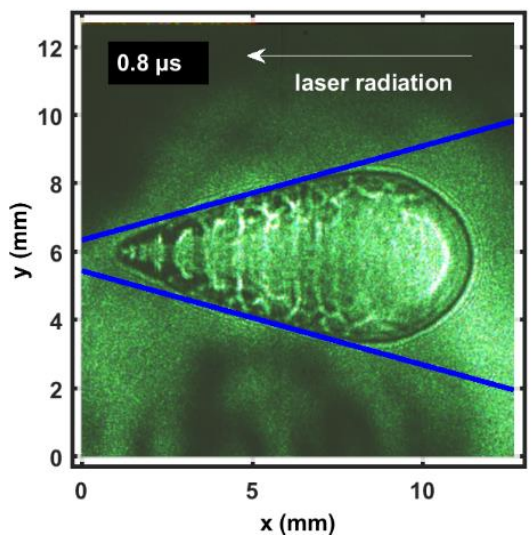

(b)

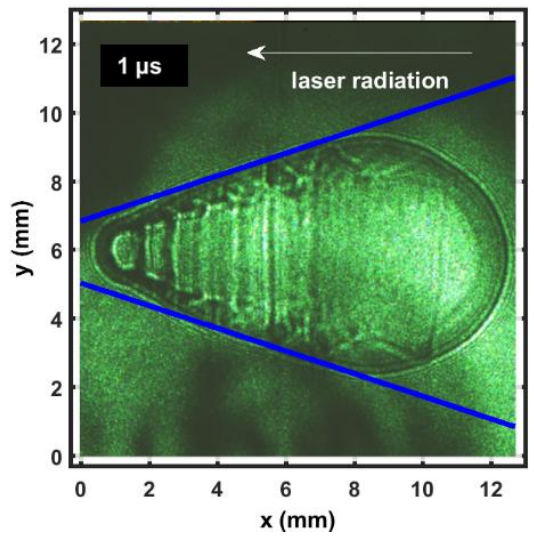

Figure 2: Plasma expansion (a) at $0.8 \mu \mathrm{s}$ (b) at $1 \mu \mathrm{s}$. At $1 \mu \mathrm{s}$ time delay, the plasma expands vertically at $\sim$ Mach 3 $(\sim 1 \mathrm{~km} / \mathrm{s})$. The slopes in (a) and (b) are \pm 3.6 and \pm 3 , respectively, indicate the forward envelopes of the shock wave.

The laser-supported plasma expansion is consistent with previous focal volume investigations ${ }^{21}$ and with the Taylor-Sedov blast wave model ${ }^{22}$. Increased electron density and temperature occur in the outer region of the plasma kernel as evidenced by the bright-to-dark boundaries that appear to cause multiple reflection inside the shock wave. The vertical extend is about a factor of 1.4 smaller for $150 \mathrm{~mJ}$ pulses than that for $850 \mathrm{~mJ}$ pulses, according to the Taylor-Sedov energy ${ }^{1 / 5}$ dependency for the radius of a spherical expansion.

\section{RESULTS AND DISCUSSION}

The experimental series for the separate measurements of atomic $\mathrm{H}$ and $\mathrm{CN}$ molecular distribution after optical breakdown includes evacuating the cell to a nominal mercury pump vacuum of $10^{-4} \mathrm{~Pa}\left(10^{-6}\right.$ Torr) and then introducing hydrogen or the $\mathrm{N}_{2}: \mathrm{CO}_{2}$ mixture. Figure 3 illustrates typical raw images of captured time-resolved data following optical breakdown ${ }^{14}$ in the ultra-high pure $\mathrm{N}_{2}$ and research grade $\mathrm{CO}_{2}$.

(a)

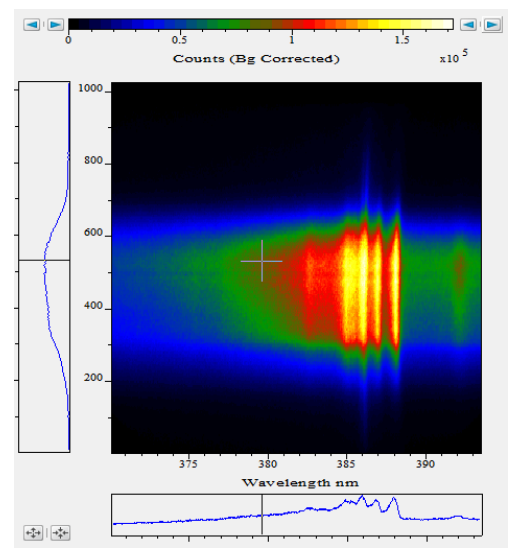

(b)

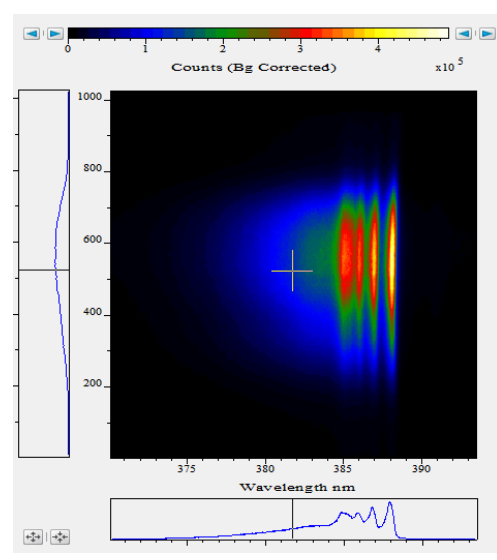

Figure 3: Raw spectra (a) $450 \mathrm{~ns}$ and (b) $3700 \mathrm{~ns}$ after optical breakdown in a 1:1 $\mathrm{CO}_{2}: \mathrm{N}_{2}$ atmospheric gas mixture. 
In the reported investigations, signatures of the $0-0,1-1,2-2,3-3,4-4$ band heads begin to emanate for time delays of the order of $100 \mathrm{~ns}$ from optical breakdown. Moreover, the plasma typically propagates towards the laser side. The recorded data indicate a $\sim 0.8 \mathrm{~mm}$ upward $\mathrm{CN}$-signal propagation in the 370 to $393.5 \mathrm{~nm}$ spectral, $7 \mathrm{~mm}$ object window during the first 5 microseconds, from a delay of $200 \mathrm{~ns}$ to $5200 \mathrm{~ns}$. Optical breakdown inside the chamber occurs at a rate of $10 \mathrm{~Hz}$, with the laser beam focused with $\mathrm{f} / 5$ optics from the top, or parallel to the slit. The detector pixels are binned in 4 tracks along the slit direction, resulting in obtaining 256 spectra for each time delay. Figure 3 shows accumulated raw data from 100 consecutive optical breakdown events, recorded at a time delay of $450 \mathrm{~ns}$ and a gate width of $125 \mathrm{~ns}$. The vertical axis indicates the slit-height, the laser beam is focused from the top. With 1:2 imaging, and a pixel resolution of $13.6 \mu \mathrm{m}$, the discernable plasma size in the cell amounts to $\sim 3 \mathrm{~mm}$. The figure shows that the $\mathrm{CN}$ band heads of the $\Delta \mathrm{v}=0$ sequence are well-developed, and it also shows an atomic line near $386.2 \mathrm{~nm}$ that likely is the carbon CI $193.09 \mathrm{~nm}$ atomic line recorded in second order.

Abel inverse transformation allows one to obtain the radial distribution of the plasma. Strictly speaking, radially symmetric profiles are required for Abel inversion. Analysis of the molecular CN spectra utilizes the same methods as previously applied for atomic hydrogen spectra ${ }^{15,16}$. The integral equation describes line-of-sight averaging,

$$
\mathrm{I}(\mathrm{z}, \lambda)=2 \int_{z}^{\rho} \mathrm{I}(r, \lambda) \frac{r}{\sqrt{r^{2}-z^{2}}} d r
$$

The measured, line-of sight data, $\mathrm{I}(\mathrm{z}, \lambda)$, along the slit dimension, $z$, are inverted for each wavelength, $\lambda$, to obtain the radial intensity distribution, $\mathrm{I}(r, \lambda)$, with the upper limit much larger, $\rho>>R$, than the radius, $\mathrm{R}$, of the plasma. The choice of the number of Chebyshev polynomials for the inversion ${ }^{15}$ is equivalent to the use of a digital filter ${ }^{16}$ resulting in the broadening of computed radial spectra. In this work, the inversion uses 10 polynomials, a smaller number of polynomials would cause smaller spectral resolution.

Figure 4 displays results of Abel-inverted hydrogen data for a time delay of $400 \mathrm{~ns}$ from optical breakdown. For the recorded data ${ }^{13}$, the constructed Boltzmann plots utilize $\mathrm{H}_{\alpha}, \mathrm{H}_{\beta}$, and $\mathrm{H}_{\gamma}$ integrated line shapes to provide a measure for the excitation temperature distribution. Fig. 4 reveals a cooler central region and a relatively hot ring of the order of $100,000 \mathrm{~K}(8.6 \mathrm{eV})$. As indicated in the figure, the kernel expands at or near the speed of sound in hydrogen gas. Fig. 5 shows the corresponding electron densities. The error bars are in part due to the lower fidelity of the Abel inversion for a time delay of $400 \mathrm{~ns}$.

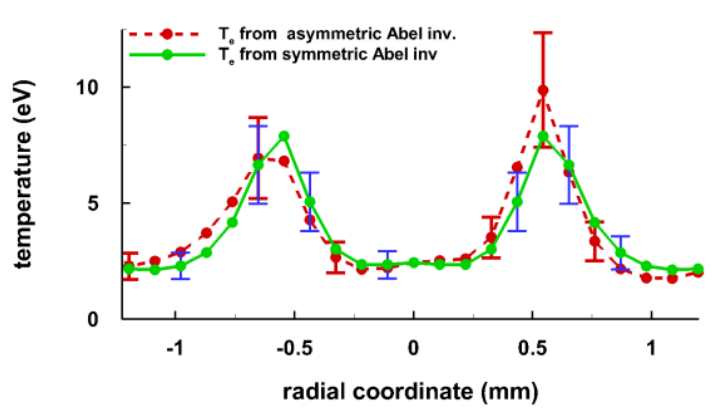

Figure 4: Electron temperature, $\mathrm{T}_{\mathrm{e}}$, vs. radial position.

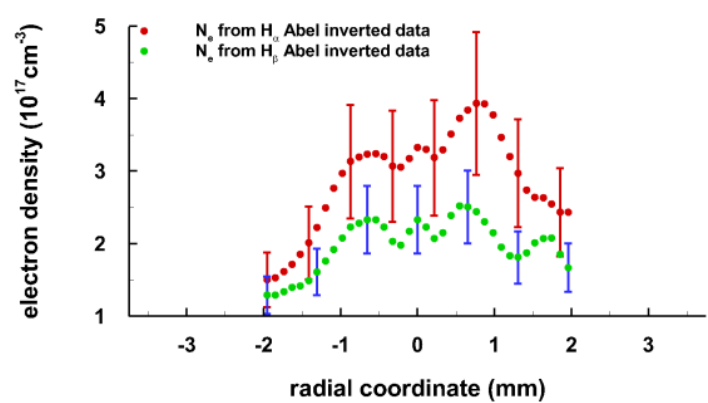

Figure5: Electron density, ne, vs. radial position. 
Wavelength and detector system-sensitivity calibrated $\mathrm{CN}$ spectral data are Abel inverted ${ }^{14}$. Figure 6 displays the results and it shows a comparison of computed and of measured spectra ${ }^{23,24}$. Similar to recently reported hydrogen nitrogen gas mixtures ${ }^{25}$, the $\mathrm{CN}$ signals are weaker at the center and indicate a slightly lower temperature than for the $0.85 \mathrm{~mm}$ position. There appears to be residual interference from an atomic line at $386.2 \mathrm{~nm}$ that likely indicates the carbon line at $193.09 \mathrm{~nm}$ line measured in second order.

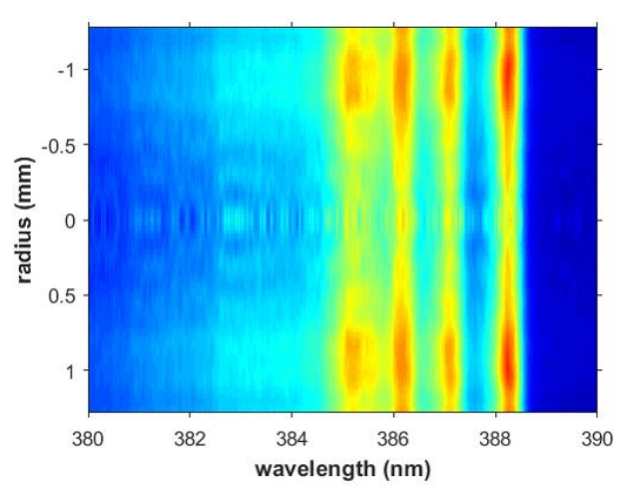

Figure 6: Abel inverted spectra versus radius at $1200 \mathrm{~ns}$.

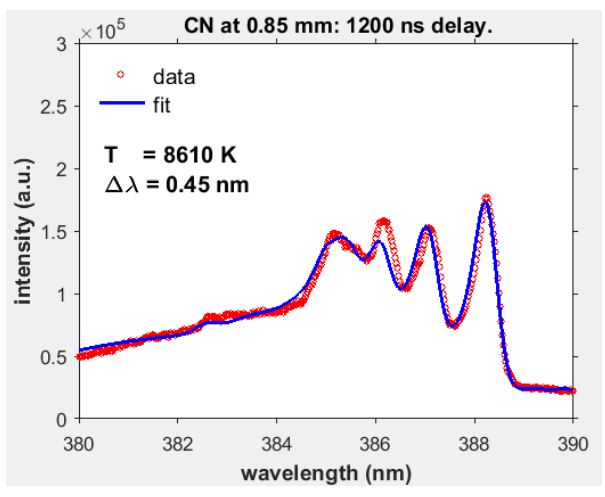

Figure 7: Inferred CN excitation temperature at $0.85 \mathrm{~mm}$.

For comparison, reasonable signal to noise spectra at $1 \mathrm{~mm}$ and at a delay of $3700 \mathrm{~ns}$, reveal expected lower temperature than that for the $1200 \mathrm{~ns}$ time delay. One would expect that the $\mathrm{CN}$ molecule distribution is close to uniform in chemical equilibrium. For time delays in the range of 5 to $50 \mu \mathrm{s}$, the line-of-sight molecular $\mathrm{CN}$ spectra are well developed, and the recorded optical emissions originate from a decreasing volume with increasing gate delay. In addition, $\mathrm{CN}$ recombination radiation signals may be stronger for specific ranges of temperature. Computation of the freely available Chemical Equilibrium with Applications (CEA) code ${ }^{19}$ elucidate CN mass fractions versus temperature. Several atoms and molecules including ionic species are part of the CEA computations, but the results for the $\mathrm{CN}$ mass fractions are of primary interest in this work. Figure 8 shows the $\mathrm{CN}$ distribution for both air and the mixture as function of temperature.

(a)

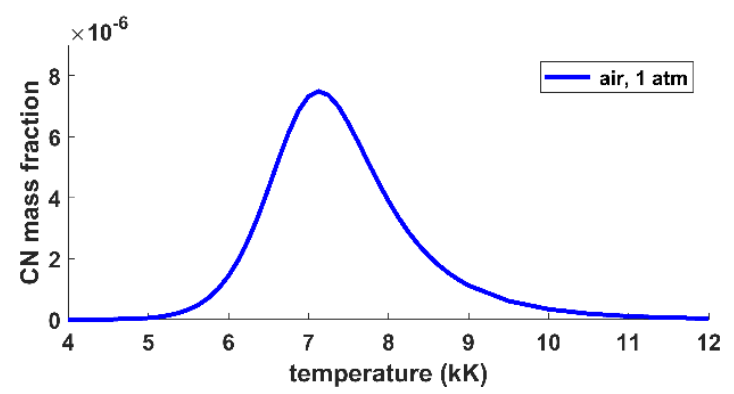

(b)

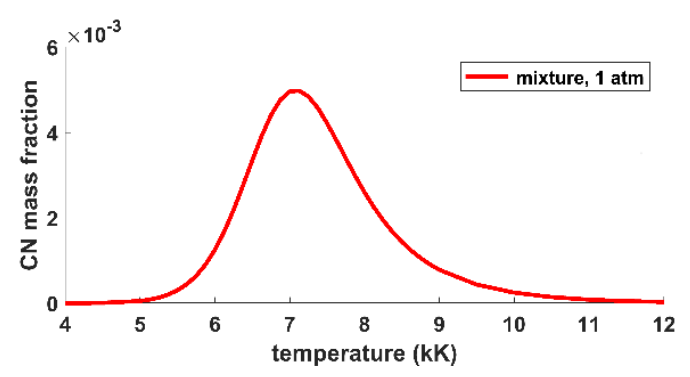

Figure 8: $\mathrm{CN}$ (a) air and (b) mixture mass fraction vs. temperature in chemical equilibrium, $\mathrm{CN}$ shows a maximum near $7 \mathrm{kK}$ for the 1:1 atmospheric $\mathrm{CO}_{2}: \mathrm{N}_{2}$ mixture. $\mathrm{CN}$ fractions in air are nearly 3 orders of magnitude lower than that for the mixture.

The recombination signals from the mixture are strongest near 7,000 K. Using the tabulated chemical equilibrium results ${ }^{19}$ at a temperature of $7 \mathrm{kK}$, one finds that the number of $\mathrm{CN}$ molecules in the mixture and air amount to $\sim 3 \times 10^{15} \mathrm{~cm}^{-3}$ and $\sim 5 \times 10^{12} \mathrm{~cm}^{-3}$, respectively. Spectra analyses of the central region results at $450 \mathrm{~ns}$ time delay reveal slightly lower temperatures at center than that obtained at a radial position of $1 \mathrm{~mm}$, and spectroscopic interference from the likely carbon line in $2^{\text {nd }}$ order is apparent for time delays of 450 and $1200 \mathrm{~ns}$. 
Line-of-sight data consist of average spectra with contributions from regions at different temperatures. For instance, the molecular $\mathrm{CN}$ spectra near the plasma edges, viz. near the top and bottom of the spectra (see Figure 3) show smaller signals but reveal higher temperatures than in the center portion.

\section{CONCLUSIONS}

Measured hydrogen and cyanide recombination spectra indicate a spherical shell structure of the plasma kernel inside the shock wave early in the plasma decay. For hydrogen, electron density and excitation temperature are higher in the peripheral region than near the center due to expansion dynamics of the plasma kernel after optical breakdown. The recombination radiation from $\mathrm{CN}$ shows a similar trend for early time delays, namely, higher excitation temperatures occur in the outer region than near the center of the plasma kernel. Strictly speaking, the application of Abel inversion requires a symmetric light source, yet the analysis of the extent of asymmetry in the hydrogen plasma leads to variations within the estimated error bars. Shadow-graph studies in air would support symmetrizing the spectral data recorded in the $\mathrm{CO}_{2}: \mathrm{N}_{2}$ mixture and applying Abel inverse transforms to extract the spatial variation of the $\mathrm{CN}$ optical emission signals.

\section{REFERENCES}

[1] D.A. Cremers, L.J. Radziemski, Handbook of Laser-Induced Breakdown Spectroscopy, Wiley \& Sons Ltd, USA, 2006.

[2] J.P. Singh, S.N. Thakur (Eds.), Laser Induced Breakdown Spectroscopy, Elsevier, NL, 2007.

[3] D. Hahn, N. Omenetto, Appl. Spectrosc. 66 (2012) 437.

[4] C.G. Parigger, D.M. Surmick, G. Gautam, A.M. EL Sherbini, Opt. Lett. 40 (2015) 3436.

[5] C.G. Parigger, Laser-induced breakdown in gases: experiments and simulation, chapter 4 in: A.W. Miziolek, V. Palleschi, I. Schechter (Eds.), Laser Induced Breakdown Spectroscopy, Cambridge University Press, USA, 2006.

[6] M. Dong, J. Lu, S. Yao, Z. Zhong, Junyan Li, Jun Li, W. Lu, Opt. Express 19 (2011) 17021.

[7] M. Dong, G.C.-Y. Chan, X. Mao, J.J. Gonzalez, J. Lu, R.E. Russo, Spectrochim. Acta Part B 100 (2014) 62.

[8] M. Kotzagianni, S. Couris, Chem. Phys. Lett. 561-562 (2013) 36.

[9] P.V. Minorsky, Plant Physiol. 155 (2011) 169.

[10] M. Baudelet, L. Guyon, J. Yu, J.-P. Wolf, T. Amodeo, E. Fréjafon, P. Laloi, J. Appl. Phys. 99 (2006) 063901.

[11] M. Baudelet, L. Guyon, J. Yu, J.-P. Wolf, T. Amodeo, E. Fréjafon, P. Laloi, J. Appl. Phys. 99 (2006) 084701.

[12] J. Ma, P.K. Dasgupta, Anal. Chim. Acta 673 (2010) 117.

[13] C.G. Parigger, D.M. Surmick, G. Gautam, J. Phys.: Conf. Ser. 810 (2017) 012012.

[14] C.G. Parigger, C.M. Helstern, G. Gautam, Int. Rev. At. Mol. Phys. 8 (2017) 25.

[15] G. Pretzler, Z. Naturforsch. 46a (1991) 639-641.

[16] G. Pretzler, H. Jäger, T. Neger, H. Philipp, and J. Woisetschläger, Z. Naturforsch. 47a (1992) 955.

[17] C.G. Parigger, G. Gautam, D.M. Surmick, Int. Rev. At. Mol. Phys. 6 (2015) 43.

[18] S. Eschlböck-Fuchs, A. Demidov, I. Gornushkin, T. Schmid, R. Rössler, N. Huber, U. Panne, J. Pedarnig, Spectrochim. Acta Part B 123 (2016) 59.

[19] B.J. McBride, S. Gordon, Computer Program for Calculating and Fitting Thermodynamic Functions, NASA RP-1271, 1992; on-line 2005 version at https://cearun.grc.nasa.gov/ (last accessed November 26, 2017).

[20] G. Gautam, C.M. Helstern, K.A. Drake, C.G. Parigger, Int. Rev. At. Mol. Phys. 7 (2016) 45.

[21] Y.-L. Chen, J.W.L. Lewis, C. Parigger, J. Quant. Spectrosc. Radiat. Transf. 67 (2000) 91.

[22] G.I. Taylor, Proc. Roy. Soc. A 201 (1950) 175.

[23] J.O. Hornkohl, C. Parigger, J.W.L. Lewis, J. Quant. Spectrosc. Radiat. Transf. 46 (1991) 405.

[24] C.G. Parigger, A.C. Woods, D.M. Surmick, G. Gautam, M.J. Witte, J.O. Hornkohl, Spectrochim. Acta Part B 107 (2015) 132.

[25] G. Gautam, C.G. Parigger, C.M. Helstern, K.A. Drake, Appl. Opt. 33 (2017) 9277. 